\title{
Proanthocyanidins inhibit Ascaris suum glutathione-S-transferase activity and increase susceptibility of larvae to levamisole and ivermectin in vitro
}

Article

Accepted Version

Creative Commons: Attribution-Noncommercial-No Derivative Works 4.0

Hansen, T. V. A., Fryganas, C., Acevedo, N., Carballo, L. R., Thamsborg, S. M., Mueller-Harvey, I. and Williams, A. R. (2016) Proanthocyanidins inhibit Ascaris suum glutathione-Stransferase activity and increase susceptibility of larvae to levamisole and ivermectin in vitro. Parasitology International, 65 (4). pp. 336-339. ISSN 1873-0329 doi:

https://doi.org/10.1016/j.parint.2016.04.001 Available at https://centaur.reading.ac.uk/62982/

It is advisable to refer to the publisher's version if you intend to cite from the work. See Guidance on citing.

To link to this article DOI: http://dx.doi.org/10.1016/j.parint.2016.04.001

Publisher: Elsevier

All outputs in CentAUR are protected by Intellectual Property Rights law, including copyright law. Copyright and IPR is retained by the creators or other copyright holders. Terms and conditions for use of this material are defined in the End User Agreement. 


\section{www.reading.ac.uk/centaur}

\section{CentAUR}

Central Archive at the University of Reading

Reading's research outputs online 
Proanthocyanidins inhibit Ascaris suum glutathione-S-transferase activity and increase susceptibility of larvae to levamisole in vitro.

5

6 Tina V.A. Hansen ${ }^{1}$, Christos Fryganas ${ }^{2}$, Nathalie Acevedo ${ }^{3}$, Luis Caraballo ${ }^{3}$, Stig M. Thamsborg ${ }^{1}$, Irene

$7 \quad$ Mueller-Harvey ${ }^{2}$, Andrew R. Williams ${ }^{1 *}$

8

$9 \quad{ }^{1}$ Department of Veterinary Disease Biology, Faculty of Health and Medical Sciences, University of

Copenhagen, Frederiksberg, Denmark.

$11{ }^{2}$ Chemistry and Biochemistry Laboratory, School of Agriculture, Policy and Development, University of

Reading, Reading, United Kingdom.

${ }^{3}$ Institute for Immunological Research, University of Cartagena, Cartagena, Colombia.

*arw@sund.ku.dk 

6

\section{Abstract}

Proanthocyanidins (PAC) are a class of plant secondary metabolites commonly found in the diet that have shown potential to control gastrointestinal nematode infections. The anti-parasitic mechanism(s) of PAC remain obscure, however the protein-binding properties of PAC suggest that disturbance of key enzyme functions may be a potential mode of action. Glutathione-S-transferases (GSTs) are essential for parasite detoxification and have been investigated as drug and vaccine targets. Here, we show that purified PAC strongly inhibit the activity of both recombinant and native GSTs from the parasitic nematode Ascaris suum. As GSTs are involved in detoxifying xenobiotic substances within the parasite, we hypothesised that this inhibition may render parasites hyper-susceptible to anthelmintic drugs. Migration inhibition assays with A. suum larvae demonstrated that the potency of levamisole (LEV) and ivermectin (IVM) were significantly increased in the presence of PAC purified from pine bark (4.6-fold and 3.2-fold reduction in IC 50 value for LEV and IVM, respectively). Synergy analysis revealed that the relationship between PAC and LEV appeared to be synergistic in nature, suggesting a specific enhancement of LEV activity, whilst the relationship between PAC and IVM was additive rather than synergistic, suggesting independent actions. Our results demonstrate that these common dietary compounds may increase the efficacy of synthetic anthelmintic drugs in vitro, and also suggest one possible mechanism for their well-known anti-parasitic activity.

(1)

\section{(1)} 7 8 9 50 
Gastrointestinal nematodes represent a major threat to sustainable and profitable livestock production worldwide. The current reliance on a small arsenal of synthetic anthelmintic drugs has serious limitations due to the threat of drug resistance, which has already reached crisis levels in small ruminant production (Sargison, 2012), and has also been detected in nematodes of pigs and cattle (Cotter et al., 2015; Gerwert et al., 2002). A complementary approach is the identification of bioactive diets that contain natural plant compounds with anti-parasitic activity, and which can be used as nutriceuticals (Hoste et al., 2015). Such an approach may slow the threat of drug resistance by reducing the frequency of drug interventions, as well as potentially boosting the host's natural immunity (Ramírez-Restrepo et al., 2010).

Diets that are rich in proanthocyanidins (PAC - syn. condensed tannins) have been demonstrated to be effective in reducing nematode fecundity and/or burdens in a variety of livestock species (Hoste et al., 2015). Moreover, in vitro assays have confirmed that PAC have direct effects on parasite survival, with electron microscopy studies demonstrating direct physical damage to both external and internal parasite structures (Brunet et al., 2011; Williams et al., 2014). However, the mechanisms that lead to parasite death have not yet been elucidated. As PAC have a strong protein-binding affinity, interference with key enzymes is an attractive hypothesis. Consistent with this, Fakae et al. (2000) have shown that extracts from some traditional Nigerian medicinal plants inhibit the function of glutathione-S-transferases from the swine nematode Ascaris suum. This inhibition was speculated to be due to, at least in some cases, the presence of PAC. Glutathione-S-transferases play a key role in detoxification of reactive oxygen species as well as xenobiotics, and have been proposed as helminth vaccine targets (Goud et al., 2012). Thus, interference with GST function may result in endogenous toxicity to the parasite and also potentially increase the susceptibility of parasites to xenobiotics such as synthetic drugs. Indeed, Whitney et al. (2013) recently reported that ivermectin (IVM) treatment of Haemonchus contortus in lambs was more effective when the lambs consumed PAC-containing red juniper berries. 
We have previously shown that $A$. suum third-stage larvae (L3) are susceptible to the anti-parasitic activity of PAC (Williams et al., 2014). In the present study, we derived highly purified PAC from two plant sources to investigate 1) whether $A$. suum GST function was inhibited by PAC, and 2) whether exposure of $A$. suum larvae to PAC in vitro would result in synergistic increases in the efficacy of IVM and levamisole (LEV).

We first purified native $A$. suum GST (nGST) from adult worms collected from the small intestine of pigs at a local slaughterhouse (Danish Crown, Ringsted, Denmark). Worms were pulverised mechanically using liquid nitrogen and the powder was then dissolved in $15 \mathrm{~mL}$ cold Binding Buffer $(140 \mathrm{mM} \mathrm{NaCl}, 2.7 \mathrm{mM} \mathrm{KCl}, 10$ $\mathrm{mM} \mathrm{Na}_{2} \mathrm{HPO}_{4}, 1.8 \mathrm{mM} \mathrm{KH}_{2} \mathrm{PO}_{4}$ ) and centrifuged for $10 \mathrm{~min}$ at 3134g. The supernatant was filtrated through a $0.20 \mu \mathrm{m}$ syringe filter (Corning) and nGST isolated on glutathione columns (GSTrap HP ${ }^{\circledR}$, GE Healthcare) following the protocol of the manufacturer. The eluate was concentrated to $500 \mu \mathrm{L}$ and subsequently exchanged with PBS using Amicon Ultra-4 centrifugal filter units (MWCO $10 \mathrm{kDa}$ ). Protein concentration was determined by the BCA assay using BSA as a standard. In addition, recombinant GST1 (rGST1) from A. suum was produced as described elsewhere (Acevedo et al., 2013). Isolation of nGST was confirmed by coomassie stain using rGST1 as a reference. SDS-PAGE was performed in a 10\% polyacrylamide (NuPAGE ${ }^{\circledR}$ Novex ${ }^{\circledR} 10 \%$ Bis-Tris Midi Gels, Life Technologies) according to the manufacturer's recommendations except that $0.5 \mu \mathrm{L}$ DL- Dithiolthreitol (Sigma-Aldrich) was used as the reducing agent. An amount of $1.4 \mu \mathrm{g}$ nGST and $1.05 \mu \mathrm{g}$ rGST1 was applied. After electrophoresis, proteins were stained with SimplyBlue ${ }^{\mathrm{TM}}$ SafeStain (Life Technologies) for 1 hour, and visualized using Odyssey FC Imager (Li-Cor Biotechnologies). As shown if Figure 1A, nGST was successfully isolated as indicated by two bands ( 23 and $24 \mathrm{kDa}$ ) corresponding to the GST1 and GST2 isoforms previously described by Liebau et al. (1994), and consistent with the $25 \mathrm{kDa}$ single band obtained with rGST1 (Acevedo et al., 2013).

In order to test whether PAC inhibited GST function, PAC were extracted from white clover flowers (WCF; Trifolium repens) and pine bark (PB; Pinus sylvestris), purified on Sephadex-LH20 columns, and analysed by 
HPLC-MS as previously described (Gea et al., 2011; Williams et al., 2014). These plant samples were chosen as they represented the two most common classes of PAC, these being procyanidins (found in PB) and prodelphinidins (found in WCF). The second fraction to elute from the column, containing high molecular weight PAC of high purity ( $84 \%$ for PB, $100 \%$ for WCF), was used in these experiments. GST activity was assayed at $26^{\circ} \mathrm{C}$ using the GST Detection Module (GE Healthcare Life Sciences) with a final concentration of $5 \mu \mathrm{g} / \mathrm{mL}$ protein. The assay was conducted in 96 well plates and read at $340 \mathrm{~nm}$ (Spectra Max Plus 384, Molecular Devices) using 1-chloro-2,4-dinitrobenzene (CDNB, $1 \mathrm{mM}$ ) as GST substrate and reduced glutathione as the reducing agent $(0.308 \mu \mathrm{g} / \mathrm{mL}$ ). Enzyme activity (nGST) was significantly reduced in the presence of PAC (Figure 1B). Similar vales were obtained with rGST1 (data not shown). The IC $\mathrm{C}_{50}$ values were 0.96 and $0.20 \mu \mathrm{g} / \mathrm{mL}$ for PB and WCF, respectively. Thus, both procyanidin and prodelphinidin type-PAC efficiently inhibit GST activity from A. suum.

We next investigated whether exposure of $A$. suum third-stage larvae (L3) to PAC purified from PB would improve the in vitro efficacy of LEV and IVM. Pine bark PAC were chosen for these experiments as procyanidins are more commonly found in the diet than prodelphinidins. Third-stage larvae were obtained by mechanically hatching embryonated eggs as described (Williams et al., 2014). The larvae were then pretreated for 60 minutes with either 20 or $10 \mu \mathrm{g} / \mathrm{mL}$ of purified PAC, or PBS as a control. Then, concentration gradients of either LEV or IVM (both obtained from Sigma-Aldrich, Stellenbosch, Germany) were added to the PAC- or PBS-treated larvae and incubated overnight. Additional groups of larvae were incubated overnight with either PAC or PBS alone. The pre-treatment time for PAC of 60 minutes was chosen as this time-frame allows irreversible binding of PAC to A. suum larvae (A.R. Williams, unpublished data), whilst the concentrations of PAC were chosen as preliminary experiments demonstrated that they achieved approximately $15 \%$ inhibition of larval migration, thus allowing the possibility to test for synergistic effects between PAC and the synthetic drugs. Migratory ability was assessed by an agar-based assay as previously 
described (Williams et al., 2014). Inhibition of migration was expressed relative to L3 incubated in media only.

Incubation of larvae in LEV or IVM alone resulted in a dose dependent inhibition of migration (Figure 2A). For both drugs, the addition of PAC increased the efficacy, resulting in a 4.6-fold and 3.2-fold reduction in increase in efficacy represented a synergistic or additive interaction, predicted additive values for the percentage of migration inhibition were calculated from the observed inhibitory effects of the individual treatments (each concentration of drug or PAC) according to Bliss' definition of independent action (Klongsiriwet et al., 2015). The observed effect of the combined PAC/drug treatments were then compared to these calculated vales, with efficacy greater than the predicted additive effect defined as synergy. This approach demonstrated that the relationship between PAC and LEV tended to be synergistic, with consistently higher observed values for the combination than the additive values predicted by independent action (Figure 2B). The effect was particular noticeable at low concentrations of LEV and $10 \mu \mathrm{g} / \mathrm{mL}$ PAC. For IVM, the relationship was better described as additive (Figure $2 \mathrm{~B}$ ), indicating that PAC tend to enhance the activity of LEV, but in the case of IVM the two agents seem to act independently of each other to inhibit larval migration. This differential interaction of PAC with LEV and IVM is perhaps consistent with the distinct anthelmintic mechanisms of these two drugs, whereby LEV acts on nicotinic acetylcholine receptors (Sarai et al., 2015) and IVM acts by binding to glutamate-gated chloride channels (Hibbs and Gouaux, 2011).

We have thus demonstrated that $A$. suum GST function is efficiently inhibited by PAC, which may offer a mechanistic explanation to their well-documented anthelmintic activity. However, the high affinity that PAC have for proteins means it is highly unlikely that any one parasite metabolic pathway is specifically targeted. Instead, it is more plausible that a range of enzymatic functions are inhibited by PAC. In addition, 
previous studies using electron microscopy to observe nematodes exposed to PAC have noted aggregates

151

152

153

154

155

156

of material forming around the buccal cavities (Martínez-Ortíz-de-Montellano et al., 2013), and have proposed that a 'coating' effect whereby PAC form complexes with external parasite proteins leads to an inhibition of parasite feeding and subsequent mortality. Furthermore, PAC are likely to interact in vivo with both host proteins as well as the parasite, adding further complexity to the situation.

Whilst it is clear that no one single mechanism may be responsible for the anthelmintic activity of PAC, inhibition of GST function raises the possibility that the parasite's detoxification mechanisms may be impaired, which may result in increased susceptibility to drugs, or, in vivo, reactive oxygen species produced by host phagocytes. Our data suggest that the efficacy of drugs (particular LEV) may be increased when larvae are co-incubated with PAC, which is in agreement with some previous in vitro and in vivo studies involving H. contortus (Armstrong et al., 2013; Whitney et al., 2013). Further studies will be necessary to determine the mechanisms behind these combinatorial effects. Given the rapid binding of PAC to proteins (Mueller-Harvey, 2006), we speculate that in our experiments key parasite proteins were neutralised and/or destroyed during the pre-incubation with PAC, leaving the larvae more susceptible to the subsequent addition of levamisole. In addition to inhibition of GST function, other plausible mechanisms include decreased cuticle integrity due to PAC-binding, which may result in increased diffusion of drugs, and inhibition of other detoxification mechanisms such as xenobiotic efflux pumping by $p$ glycoproteins, or activity of gluconyl transferases. Thus, we cannot conclude that the synergistic effects of PAC and levamisole are due only to the GST inhibition, and the effect of PAC on the activity of these other parasite pathways is worthy of further investigation.

In conclusion, we have confirmed that PAC strongly inhibit GST function from an important parasitic nematode, and we also have demonstrated that PAC can synergistically improve the efficacy of LEV and 
174 also act additively with IVM in vitro. Further studies will focus on the mechanisms involved and whether

175 PAC-rich diets can improve drug efficacy in vivo.

176

177 Acknowledgements

178 Funding from the Danish Council for Independent Research (Technology and Production Sciences - Grant

$179 \# 12-126630)$ is gratefully acknowledged. CF was supported by the EU Marie Curie Initial Training Network

180 'LegumePlus' (PITN-GA-2011-289377). For laboratory assistance the authors are very grateful to Lars

181 Andreasen and Annie Bjergby Kristensen.

182

183

184

185

186

187

188

189

190

191

192

193

194

195

196

197

198 
Figure 1 - Isolation of Ascaris suum glutathione-S-transferase (GST) and inhibition by proanthocyanidins

A) A. suum GST was isolated from adult worms and visualised by SDS-PAGE. Lane 1 - isolated native GST, Lane 2 - recombinant GST1

B) Inhibition of native GST activity by PAC purified from pine bark (PB) and white clover flowers (WCF). Results are the mean ( \pm S.E.M) of two independent experiments, each performed in duplicate.

Figure 2 - Proanthocyanidins (PAC) increase the efficacy of levamisole and ivermectin in vitro

A) Percentage migration of Ascaris suum larvae in the presence of levamisole (LEV) and ivermectin (IVM) with or without 10 (PB10) or 20 (PB20) $\mu \mathrm{g} / \mathrm{mL}$ of PAC isolated from pine bark. Results are the mean ( \pm S.E.M) of two independent experiments, each performed in duplicate. Also shown are $\mathrm{IC}_{50}$ values calculated by non-linear regression. For each drug, values followed by different subscripts indicate significantly $(P<0.0001)$ different $\mathrm{IC}_{50}$ values.

B) Synergy analysis of levamisole (LEV) or ivermectin (IVM) combined with 10 or $20 \mu \mathrm{g} / \mathrm{mL}$ PAC from pine bark (PB). Shown is the percentage inhibition of larval migration achieved by the drug alone and in combination with PAC, and the additive values predicted by the assumption of independent action of the 


\section{References}

Acevedo, N., Mohr, J., Zakzuk, J., Samonig, M., Briza, P., Erler, A., Pomés, A., Huber, C.G., Ferreira, F., Caraballo, L., 2013. Proteomic and Immunochemical Characterization of Glutathione Transferase as a New Allergen of the Nematode Ascaris lumbricoides. PLoS ONE 8, e78353.

Armstrong, S.A., Klein, D.R., Whitney, T.R., Scott, C.B., Muir, J.P., Lambert, B.D., Craig, T.M., 2013. Effect of using redberry juniper (Juniperus pinchotii) to reduce Haemonchus contortus in vitro motility and increase ivermectin efficacy. Veterinary Parasitology 197, 271-276.

Brunet, S., Fourquaux, I., Hoste, H., 2011. Ultrastructural changes in the third-stage, infective larvae of ruminant nematodes treated with sainfoin (Onobrychis viciifolia) extract. Parasitology International 60, 419-424.

Cotter, J.L., Van Burgel, A., Besier, R.B., 2015. Anthelmintic resistance in nematodes of beef cattle in southwest Western Australia. Veterinary Parasitology 207, 276-284.

Fakae, B.B., Campbell, A.M., Barrett, J., Scott, I.M., Teesdale-Spittle, P.H., Liebau, E., Brophy, P.M., 2000. Inhibition of glutathione S-transferases (GSTs) from parasitic nematodes by extracts from traditional Nigerian medicinal plants. Phytotherapy Research 14, 630-634.

Gea, A., Stringano, E., Brown, R.H., Mueller-Harvey, I., 2011. In Situ Analysis and Structural Elucidation of Sainfoin (Onobrychis viciifolia) Tannins for High-Throughput Germplasm Screening. Journal of Agricultural and Food Chemistry 59, 495-503.

Gerwert, S., Failing, K., Bauer, C., 2002. Prevalence of levamisole and benzimidazole resistance in Oesophagostomum populations of pig-breeding farms in North Rhine-Westphalia, Germany. Parasitology Research 88, 63-68.

Goud, G.N., Deumic, V., Gupta, R., Brelsford, J., Zhan, B., Gillespie, P., Plieskatt, J.L., Tsao, E.I., Hotez, P.J., Bottazzi, M.E., 2012. Expression, purification, and molecular analysis of the Necator americanus glutathione S-transferase 1 (Na-GST-1): A production process developed for a lead candidate recombinant hookworm vaccine antigen. Protein Expression and Purification 83, 145-151.

Hibbs, R.E., Gouaux, E., 2011. Principles of activation and permeation in an anion-selective Cys-loop receptor. Nature 474, 54-60.

Hoste, H., Torres-Acosta, J.F.J., Sandoval-Castro, C.A., Mueller-Harvey, I., Sotiraki, S., Louvandini, H., Thamsborg, S.M., Terrill, T.H., 2015. Tannin containing legumes as a model for nutraceuticals against digestive parasites in livestock. Veterinary Parasitology 212, 5-17.

Klongsiriwet, C., Quijada, J., Williams, A.R., Mueller-Harvey, I., Williamson, E.M., Hoste, H., 2015. Synergistic inhibition of Haemonchus contortus exsheathment by flavonoid monomers and condensed tannins. International Journal for Parasitology: Drugs and Drug Resistance 5, 127-134.

Liebau, E., Schönberger, Ö.L., Walter, R.D., Henkle-Dührsen, K.J., 1994. Molecular cloning and expression of a cDNA encoding glutathione S-transferase from Ascaris suum. Molecular and Biochemical Parasitology 63, 167-170.

Martínez-Ortíz-de-Montellano, C., Arroyo-López, C., Fourquaux, I., Torres-Acosta, J.F.J., Sandoval-Castro, C.A., Hoste, H., 2013. Scanning electron microscopy of Haemonchus contortus exposed to tanninrich plants under in vivo and in vitro conditions. Experimental Parasitology 133, 281-286.

Mueller-Harvey, I., 2006. Unravelling the conundrum of tannins in animal nutrition and health. Journal of the Science of Food and Agriculture 86, 2010-2037.

Ramírez-Restrepo, C.A., Pernthaner, A., Barry, T.N., López-Villalobos, N., Shaw, R.J., Pomroy, W.E., Hein, W.R., 2010. Characterization of immune responses against gastrointestinal nematodes in weaned lambs grazing willow fodder blocks. Animal Feed Science and Technology 155, 99-110.

Sarai, R.S., Kopp, S.R., Knox, M.R., Coleman, G.T., Kotze, A.C., 2015. In vitro levamisole selection pressure on larval stages of Haemonchus contortus over nine generations gives rise to drug resistance and target site gene expression changes specific to the early larval stages only. Veterinary Parasitology 211, 45-53. 
Sargison, N.D., 2012. Pharmaceutical treatments of gastrointestinal nematode infections of sheep-Future of anthelmintic drugs. Veterinary Parasitology 189, 79-84.

Whitney, T.R., Wildeus, S., Zajac, A.M., 2013. The use of redberry juniper (Juniperus pinchoti) to reduce Haemonchus contortus fecal egg counts and increase ivermectin efficacy. Veterinary Parasitology 197, 182-188.

Williams, A.R., Fryganas, C., Ramsay, A., Mueller-Harvey, I., Thamsborg, S.M., 2014. Direct anthelmintic effects of condensed tannins from diverse plant sources against Ascaris suum. PLoS ONE 9, e97053. 
$281 \quad$ Figure 1

282

A

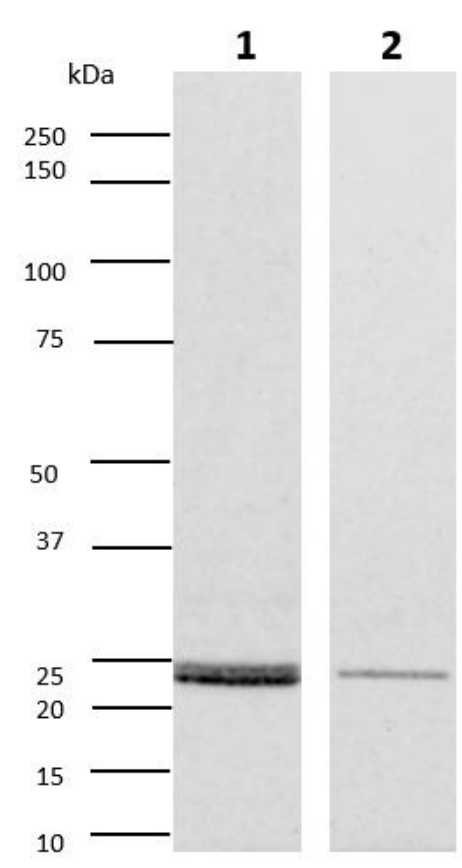

B

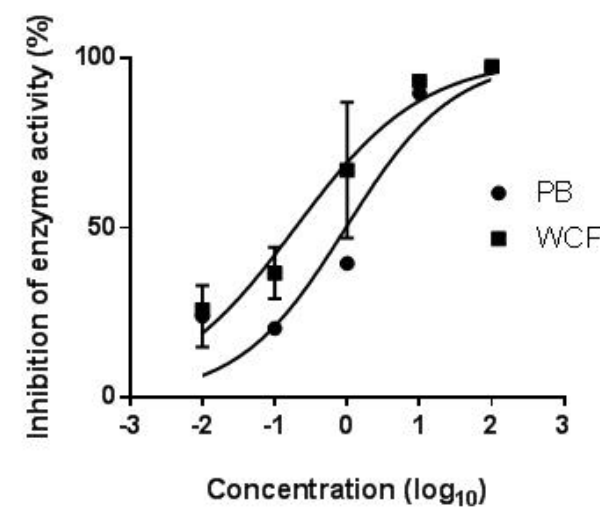


Figure 2
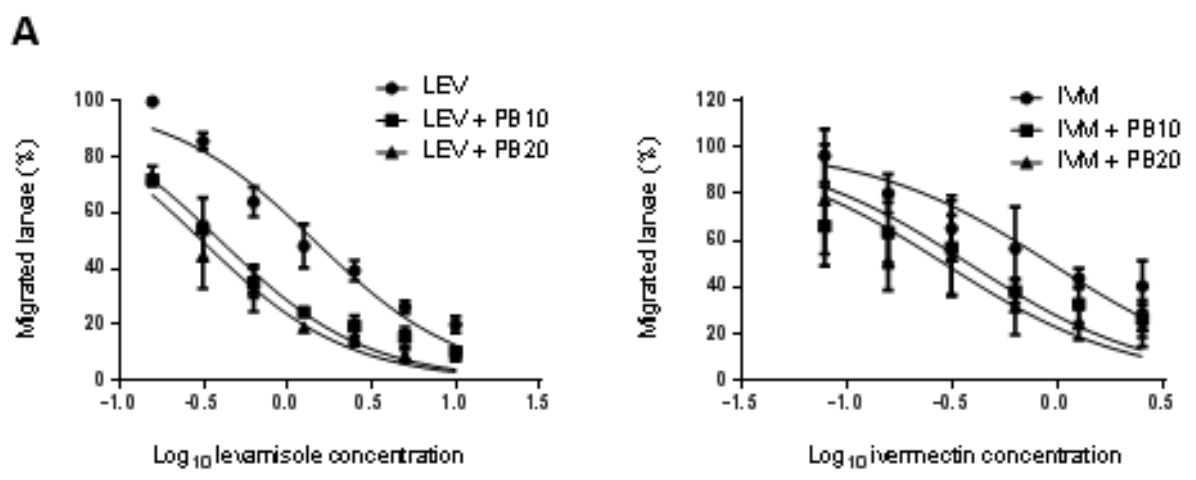

\begin{tabular}{lccc}
\hline \multicolumn{4}{c}{$\mathrm{IC}_{50}$ values $(\mu \mathrm{g} / \mathrm{mL})$} \\
\hline & Drug alone & Drug $+10 \mu \mathrm{g} / \mathrm{mL}$ PAC & Drug $+20 \mu \mathrm{g} / \mathrm{mL}$ PAC \\
\cline { 2 - 4 } Levamisole & $1.45^{\mathrm{a}}$ & $0.39^{\mathrm{b}}$ & $0.31^{\mathrm{b}}$ \\
Ivermectin & $0.93^{\mathrm{a}}$ & $0.37^{\mathrm{b}}$ & $0.29^{\mathrm{b}}$ \\
\hline
\end{tabular}

B
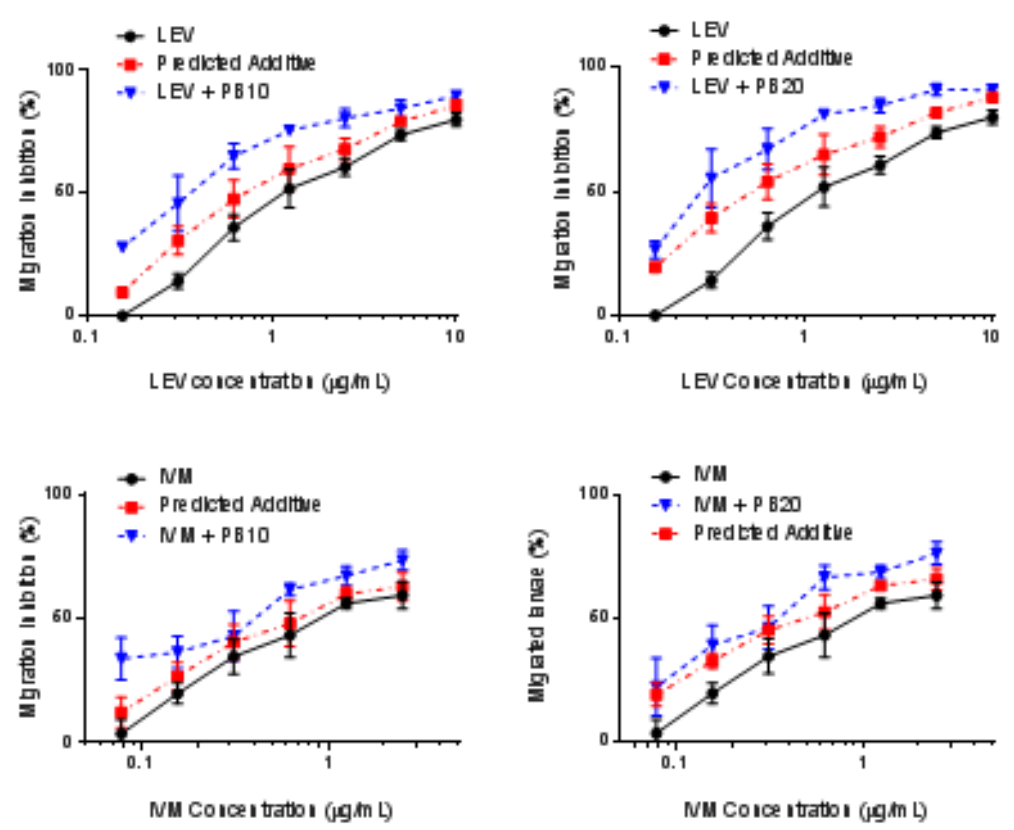